\title{
Corneal Blood Staining after Complicated Cataract Surgery
}

\author{
Mark Krauthammer Jorje Mandelblum Oriel Spierer \\ Ophthalmology Division, Tel Aviv Sourasky Medical Center, Sackler Faculty of Medicine, \\ Tel Aviv University, Tel Aviv, Israel
}

\section{Keywords}

Corneal blood staining $\cdot$ Cataract surgery $\cdot$ Intraocular pressure $\cdot$ Coumadin (warfarin sodium)

\begin{abstract}
Purpose: To report the case of a patient with corneal blood staining after a complicated cataract surgery. Observations: We report on a 68-year-old woman who developed corneal blood staining secondary to hyphema after cataract surgery, despite the fact that intraocular pressure was not increased for a prolonged time. The corneal staining spontaneously cleared from the periphery towards the center. It was fully resolved after 2.5 years of follow-up. Conclusions and Importance: In case of hyphema induced by cataract surgery, performance of early anterior chamber washout should be considered to prevent corneal blood staining and poor visual outcome.

(C) 2018 The Author(s)

Published by S. Karger AG, Basel
\end{abstract}

\section{Introduction}

Corneal blood staining is a severe ophthalmic complication that usually occurs as a result of long-standing hyphema with increased intraocular pressure (IOP). A pressure $>25 \mathrm{~mm} \mathrm{Hg}$ for $>5$ days may cause corneal staining [1]. This pathology most often occurs after a traumainduced hyphema, and the reported incidence is $2-11 \%$ of traumatic hyphema cases and even higher for total hyphemas [2]. Other corneal blood staining etiologies are hyphema induced by intraocular surgery [3] or any other bleeding in the anterior chamber (AC). In case of 
hyphema, maximal medical and surgical efforts should be applied to prevent cornea staining, since this condition may end up with an irreversible result of blurred vision due to corneal opacity [1]. Irreversible corneal staining may further lead to corneal transplantation. We herein report a case of hyphema resulting in corneal blood staining following a complicated cataract surgery.

\section{Case Report}

A 68-year-old woman, who was using warfarin sodium (Coumadin) due to atrial fibrillation, underwent a routine phacoemulsification cataract surgery on her right eye. Prior to the surgery the international normalized ratio (INR) was measured to be 3.5. During the surgery the posterior capsule was ruptured, resulting in prolapse of the vitreous into the AC. During the anterior vitrectomy, bleeding from the superior iris was seen. The bleeding was stopped using an ophthalmic viscoelastic device and intraocular diathermia. A three-piece intraocular lens (IOL) was implanted in the sulcus. On the next day, IOL haptic dislocation to the AC and a 5-mm hyphema were seen. The patient underwent repositioning of the IOL and AC washout. On the next day, a total hyphema was observed and the IOP was $26 \mathrm{~mm} \mathrm{Hg}$. Treatment with dexamethasone drops $0.1 \% 6 /$ day, ofloxacin drops $0.3 \% 4 /$ day, dorzolamide hydrochloride drops $2 \% 2$ /day, and timolol maleate drops $0.5 \% 2 /$ day was started. Further, in order to prevent corneal blood staining, latanoprost $0.005 \% 1$ dday, brimonidine tartrate $0.1 \% 2 /$ day, atropine sulfate $1 \% 1 /$ day, and Uramox $250 \mathrm{mg}$ p.o. 3/day were added. The patient was examined on a daily basis with consecutive IOPs of $18,23,30$, and $28 \mathrm{~mm} \mathrm{Hg}$. On postoperative day 6 , the hyphema occupied $90 \%$ of the AC and staining was suspected at the superior cornea. Warfarin treatment was stopped and 2 days later, when the INR had decreased to 1.48, recurrent AC washout was done. On the next day, the AC was clear of blood and total corneal staining was seen. Visual acuity (VA) was hand motion and IOP was $13 \mathrm{~mm} \mathrm{Hg}$. During further follow-up, the cornea slowly cleared from the periphery towards the center (Fig. 1a-c for postoperative months 1.5, 3, and 6, respectively), with slow but steady improvement in VA. IOPs were $12-18 \mathrm{~mm} \mathrm{Hg}$ on recurrent examinations. After 2.5 years of follow-up the cornea was completely cleared, VA had improved to 20/25, and IOP was $18 \mathrm{~mm} \mathrm{Hg}$ (Fig. 1d).

\section{Discussion}

A number of studies concluded that there is no recommendation to discontinue antiplatelet and antiaggregation agents before phacoemulsification cataract surgery [4, 5]. The hyphema in our case was probably the result of mild iris trauma caused by the ocutome during the anterior vitrectomy. This is not unexpected in a patient treated with Coumadin with a therapeutic INR value of 3 . What was unexpected was the fact that corneal staining occurred although IOP was not $>25 \mathrm{~mm} \mathrm{Hg}$ for 5 days. Few previous publications presented case reports of patients who developed corneal blood staining with low IOP. One of the reports suggested transient elevations of IOP that were not recorded during routine ambulatory followup as a reason for corneal staining [3]; in another report, endothelial dysfunction due to contusion endothelial necrosis or temporary endothelial decompensation in face of significant hyphema were suggested as possible reasons for corneal staining [6]. Apart from increased IOP, some other risk factors for development of corneal blood staining have been reported: Descemet membrane injury, endothelial damage or dysfunction, prolonged duration of 
hyphema, and large amount of blood in the AC [6]. During and after phacoemulsification cataract surgery, corneal endothelial cell function is decreased while endothelial permeability is increased. In addition, 15-18\% of the endothelial cells are lost because of the phacoemulsification energy [7]. In the current case, we speculate that the corneal endothelium cell dysfunction and loss following the cataract surgery were the main risk factors for blood penetration to the corneal stroma, resulting in early corneal staining. Although spontaneous resolution of the staining may occur, many patients end up with permanent corneal opacity and a need for corneal transplantation [1]. As far as we know, no large-scale studies about either spontaneous resolution of corneal staining or nonsurgical treatment options have been reported.

To the best of our knowledge, this is the first report of corneal staining after cataract surgery. The staining, which occurred in a patient who developed total hyphema with intermittent and only mild elevation of IOP levels, was probably associated with a temporary corneal endothelial layer dysfunction. We recommend that in case of total hyphema after a phacoemulsification cataract surgery, an early surgical intervention for AC washout be considered in order to avoid corneal blood staining.

\section{Statement of Ethics}

The patient provided oral consent for publication of this report.

\section{Disclosure Statement}

The authors have no financial disclosures. This work received no financial support.

\section{References}

1 Weisenthal RW, Afshari NA, Bouchard CS, Colby KA, Rootman DS, Tu EY, et al. Basic and clinical science course. External disease and cornea. American Academy of Ophthalmology 2015, Section 8, p. 353-4.

2 Walton W, Von Hagen S, Grigorian R, Zarbin M. Management of traumatic hyphema. Surv Ophthalmol. 2002 Jul-Aug;47(4):297-334.

3 Fraser C, Liew S, Fitzsimmons R, Arnold J. Spontaneous resolution of corneal blood staining. Clin Experiment Ophthalmol. 2006 Apr;34(3):279-80.

4 Barequet IS, Sachs D, Priel A, Wasserzug Y, Martinowitz U, Moisseiev J, et al. Phacoemulsification of cataract in patients receiving Coumadin therapy: ocular and hematologic risk assessment. Am J Ophthalmol. 2007 Nov;144(5):719-23.

5 Jamula E, Anderson J, Douketis JD. Safety of continuing warfarin therapy during cataract surgery: a systematic review and meta-analysis. Thromb Res. 2009 Jul;124(3):292-9.

6 Beyer TL, Hirst LW. Corneal blood staining at low pressures. Arch Ophthalmol. 1985 May;103(5):654-5.

7 Díaz-Valle D, Benítez Del Castillo Sanchez JM, Toledano N, Castillo A, Pérez-Torregrosa V, García-Sanchez J. Endothelial morphological and functional evaluation after cataract surgery. Eur J Ophthalmol. 1996 Jul-Sep; $6(3): 242-5$. 


\section{Case Reports in Ophthalmology}
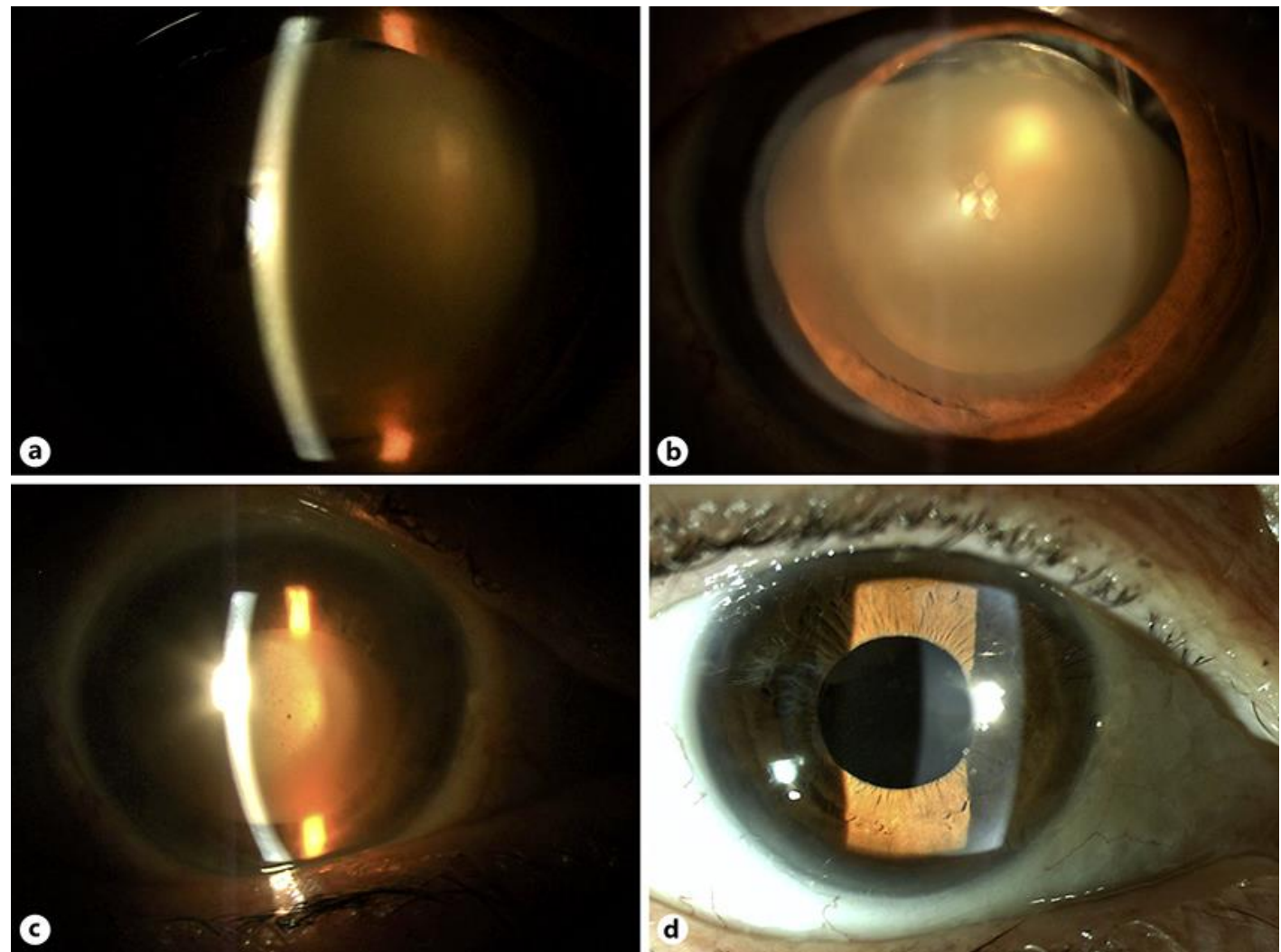

Fig. 1. The course of spontaneous resolution of the corneal staining. a Six weeks after surgery. b Three months after surgery. $\mathbf{c}$ Six months after surgery. $\mathbf{d}$ Two and a half years after surgery. 